\title{
Screening of Tomato Varieties for Resistance to Tomato Leaf Curl Viral Disease under Field Conditions
}

\author{
Sajad Ali Solangi ${ }^{1}$, Jamal-U-Ddin Hajano ${ }^{1 *}$, Rehana Naz Syed ${ }^{1}$, Sohail Ahmed Otho ${ }^{2}$, Khadim Hussain \\ Wagan', Agha Mushtaque Ahmed $^{2}$, Fahad Nazir Khoso' , Aftab Raza Jarwar ${ }^{3}$ and Suman Tarique Qazi ${ }^{1}$
}

${ }^{1}$ Department of Plant Pathology, Faculty of Crop Protection, Sindh Agriculture University, Tandojam 70060, Pakistan; ${ }^{2}$ Department of Entomology, Faculty of Crop Protection, Sindh Agriculture University, Tandojam 70060, Pakistan; ${ }^{3}$ Department of Plant Protection, Faculty of Crop Protection, Sindh Agriculture University, Tandojam 70060, Pakistan.

Abstract | Tomato (Lycopersicum esculentum Mill.) is important vegetable in Pakistan. Tomato leaf curl viral disease caused by tomato leaf curl virus (TLCV) (genus: Begomovirus, family: Geminiviridae) and transmitted by whitefly (Bemisia tabaci) is considered as the most devastating one. Recently, available tomato varieties are not screened against this disease in the agro-ecosystem of Sindh Province of Pakistan. Therefore, in this study 12 commercial varieties of tomato such as, TO-1057, Advanta-1225, T-1359, Yaqui, Sahil, Ayushman, Early king, Advanta-1211, Kiara, Lima, Advanta-1209 and Ashoka were screened against the disease under field conditions during Rabi and Kharif growing season 2017-18. Observations were taken on disease incidence, severity and whiteflies population at interval of week after date of transplantation. Additionally, relationship among the disease and vector insect was determined. The minimum incidence of the disease was recorded in variety Advanta-1211 (13.85\%) and Lima (18.85\%) followed by T-1359 (23.30\%), Early king (25.51\%), TO1057 (27.21\%), Advanta-1225 (29.41\%). Minimum 1-rating score was recorded in Advanta-1211, Lima and T-1359 varieties. Significantly minimum number of whiteflies was recorded in variety Lima (2.3 whiteflies), Advanta-1209 (2.3 whiteflies), Kiara (2.4 whiteflies) followed by Advanta-1211 (2.5 whiteflies). There was non-significant relationship of vector insect with both incidence as well severity of tomato leaf curl disease, $r^{2}=0.07068, P=0.8272$ and $r^{2}=0.2869, P=0.3660$, respectively. Based on the results, it is concluded that the minimum incidence and severity of tomato leaf curl disease was recorded in variety Advanta-1211, Lima and $\mathrm{T}$-1359. In rabi season the disease intensity was lower as compare to kharif season. Therefore, it is suggested that variety Advanta-1211, Lima and T-1359 should incorporated in the disease management plan.

Received | April 30, 2021; Accepted | February 03, 2022; Published | February 16, 2022

*Correspondence | Jamal-U-Ddin Hajano, Department of Plant Pathology, Faculty of Crop Protection, Sindh Agriculture University, Tandojam 70060, Pakistan; Email: hajanojamal@gmail.com; jhajano@sau.edu.pk

Citation | Solangi, S.A., J.U.D. Hajano, R.N. Syed, S.A. Otho, K.H.Wagan, A.M. Ahmed, F.N. Khoso, A.R. Jarwar and S.T. Qazi. 2022. Screening of tomato varieties for resistance to tomato leaf curl viral disease under field conditions. Pakistan Journal of Agricultural Research, 35(1): 70-77. DOI | https://dx.doi.org/10.17582/journal.pjar/2022/35.1.70.77

Keywords | Tomato, Tomato leaf curl viral disease, Resistance screening, Whitefly (Bemisia tabaci) and varieties

\section{Introduction}

$\mathrm{T}$ omato (Lycopersicume esculentum Mill.) belongs to the family Solanaceae and is a popular grown vegetable and rank third of worldwide vegetable production (Sadaf et al., 2012). Tomato is a good source of vitamins (A and $\mathrm{C}$ ) and minerals. It is one of the most widely grown vegetable crops, highly popular due to its high nutritive value, taste and versatile use in various foods. In Pakistan tomato crop is cultivated on 58.4 thousand hectares, with production of 551.0 thousand tonnes and per hectare yield is estimated 
9.4 thousand tonnes (GoP, 2018). However, tomato crop in Sindh is grown on area of 25.0 thousand hectares which produced 182.2 thousand tonnes with average yield per hectare is 7.3 thousand tonnes $(\mathrm{GoP}$, 2018). Sindh province ranks first in Pakistan with reference to area under cultivation and total production (GoP, 2018). However, yield per hectare is lower as compare to Punjab, Balochistan and KPK province, respectively (GoP, 2018). Tomato production is reducing due to abiotic and biotic factors which prerequisite to boost up crop yield in Pakistan. Among biotic factors, plant viruses attract considerable attention for vegetable production because they impose significant production constraints. Tomato crop is susceptible to approximately 136 viruses under natural or artificial conditions (Dallwitz, 1980; Dallwitz et al., 1993; Brunt et al., 1996). Viral diseases of tomatoes are causing serious damages and large economic losses. The amount of loss depends on the viral disease involved, the strain of the virus, the variety of tomato, the age of the plant at infection time, the presence of other diseases, and the extent that viruses have spread during the planting of the crop (Walkey, 1985).

Approximately 13 plant viruses are known to infect tomato crop in Pakistan. Most of tomatoes infecting viruses are predominantly transmitted by white fly, aphids and some are mechanically transmitted (Hassan and Thomas, 1985; Hassan et al., 1993). Among these viral pathogens, tomato leaf curl virus (TLCV, genus: Begomovirus, family: Geminiviridae) causing tomato leaf curl viral disease (TLCVD) is considered as the most devastating one (Nono-womdim, 2001; Zeshan et al., 2016). TLCV is consisting on ssDNA $(+/-)$ genome and two incomplete icosahedral geminate particles. Up to $100 \%$ yield losses in tomato crop are documented due to this problem (Shelat et al., 2014). Whitefly (Bemisia tabaci) has been shown to transmit TLCV in persistent circulative manner (Zeshan et al., 2016).

Such viral pathogens could be controlled by the use of chemical against the vector insects, but the chemical can create hazards to human health and can produce undesirable side effects on non-targeted organisms. Although development of resistant varieties is time consuming strategy but is the most effective method of controlling many viral diseases. Additionally, this strategy is easy to and cost effective. Therefore, available varieties are screened on regular basis to assess their potential to specific viral pathogen. For such purpose previously, Hameed and Khalid (1996) collected tomato varieties from AVRDC for screening resistance to TLCV, only Tyking (a Hybrid cultivar), L. hirsutum (LA-1777) and L. chilenese (LA-1969) are resistant to the virus. Similarly, Zeshan et al. (2016) screened twenty-seven tomato varieties/lines against TLCVD, number of highly susceptible, susceptible, moderately susceptible, moderately resistant and resistant were three, six, four, six and eight, respectively. Highly resistant and immune varieties/lines against TLCVD were not found in the study. However, with passage of time and change in ecological conditions resistance equilibrium in varieties can fluctuate therefore there is needed to assess tomato varieties. Purpose of the study was to determine the resistance potential of different commercially grown tomato varieties against TLCVD in agro-ecological zone of Sindh and to analyze the relationship between the disease intensity with vector population.

\section{Materials and Methods}

\section{Experimental sites}

Experiments were conducted in open field at vicinity of Tandojam.

\section{Planting material and experimental design}

Twelve commercial varieties of tomato were tested against the TLCVD under natural inoculation conditions (Table 1). Trials were conducted in rabi (winter) and kharif (summer) seasons during 2017 and 2018 , respectively. A total 27 plants of each variety were grown with three replications (9 per repeat, 3 repeats $=27$ plants $)$ in complete randomize block design in same experimental plot.

\section{Table 1: Details of tomato varieties used in this study.}

$\begin{array}{lll}\text { S. No. } & \text { Variety } & \text { Distributer } \\ 1 & \text { TO-1057 } & \text { Syngenta } \\ 2 & \text { Advanta } 1225 & \text { ICI } \\ 3 & \text { T-1359 } & \text { Syngenta } \\ 4 & \text { Yaqui } & \text { Seminis } \\ 5 & \text { Sahil } & \text { Syngenta } \\ 6 & \text { Ayushman } & \text { Seminis } \\ 7 & \text { Early king } & \text { Millan Agro Seed } \\ 8 & \text { Advanta } 1211 & \text { ICI } \\ 9 & \text { Kiara } & \text { Siddique sons } \\ 10 & \text { Lima } & \text { Haji sons } \\ 11 & \text { Advanta } 1209 & \text { ICI } \\ 12 & \text { Ashoka } & \text { Seminis }\end{array}$

March 2022 | Volume 35 | Issue 1 | Page 71 
Observation and measurements

Observations were taken on the disease incidence, severity and whiteflies population at interval of week after date of transplantation. Percent of the disease incidence was determined by dividing number of tomato plants showing leaf curling symptoms with total number of plants examined for the disease. A 0-5 rating scale was used to measure the disease severity (Zeshan et al., 2016) where, 0 is indicating all plants free from disease symptoms, $1=1-20 \%$ infection, $2=$ $21-40 \%$ infection, $3=41-60 \%$ infection, $4=61-80 \%$ infection and 5 $=81-100 \%$ infection. Whiteflies population (both adults \& nymphs) was recorded from three randomly selected plants of each replication at upper leaf (first plant), middle leaf (second plant), and lower leaf (third plant) during the time of visual disease assessment (Perveen et al., 2010; Akram et al., 2013). Each plant was rated and then averaged in the group of 9 plants to represent one replication and there were three such replications of that variety.

\section{Statistical analysis}

A multifactorial ANOVA was performed to analyze difference in the disease incidence and whiteflies in interaction of varieties $\times$ season $\times$ Time of assessment using LSD test $(\alpha=0.05)$ of completely randomized design using STATISTIX v. 8.1 software (Analytical Software). Nonparametric spearman test at $95 \%$ confidence interval was used to analysis correlation of the disease incidence and severity with vector population.

\section{Results and Discussion}

Incidence of the tomato leaf curl disease in different tomato varieties

The incidence of TLCVD was recorded in different commercial tomato varieties under natural inoculum in field conditions during rabi and kharif growing season 2017-18. On average the minimum incidence of the disease was recorded in variety Advanta-1211 (13.85\%) and Lima (18.85\%) followed by T-1359 (23.30\%), Early king (25.51\%), TO-1057 (27.21\%), Advanta-1225 (29.41\%) (Figure 1). Similarly, average maximum incidence of TLCVD was recorded in variety Ayushman (51.13\%) and Ashoka (45.07\%) followed by Advanta-1209 (40.01\%), Kiara (38.38\%), Yaqui (36.17 \%) and Sahil (32.77\%) (Figure 1). Variety Advanta-1211 showed significantly lower incidence $6.60 \%$ of the disease during rabi growing season followed by TO-1057 (9.90\%), Early king (14.30 $\%)$ and T-1359 (12.10\%), whereas, during same growing season Ashoka variety showed maximum incidence of the disease $40.0 \%$ followed by Ayushman (31.13\%), Kiara (30.07\%) and Advanta-1225 (21.10 \%) (Table 2). During Kharif growing season, minimum tomato leaf curling was recorded in Advanta-1211 (21.10\%) followed by T-1359 (34.50\%), Early king (36.73 \%) and Advanta-1225 (37.73\%), while maximum incidence of the disease during kharif season was recorded in Ayushman variety (71.13 \%) followed by Ashoka (50.13 \%), Sahil (47.83 \%), Kiara (46.70 \%) and TO-1057 (44.53 \%) (Table 2). Generally, the disease incidence was higher in kharif as compare to rabi season but in variety Yaqui, Lima and Advanta-1209 the situation was vice versa. Variety Lima showed 9.90 and $27.80 \%$ leaf curling in kharif and rabi season, respectively (Table 2). There was non significant variation in the disease incidence $\%$ recorded in Yaqui variety even it was higher (36.67 \%) during rabi season as compare to kharif (35.57 \%) (Table 2). Similarly, Advanta-1209 variety showed higher incidence in the rabi season and lower (38.87 $\%$ ) in kharif growing season (Table 2). However, variety Lima showed minimum incidence of curling symptoms during both growing seasons as compare to other tested varieties.

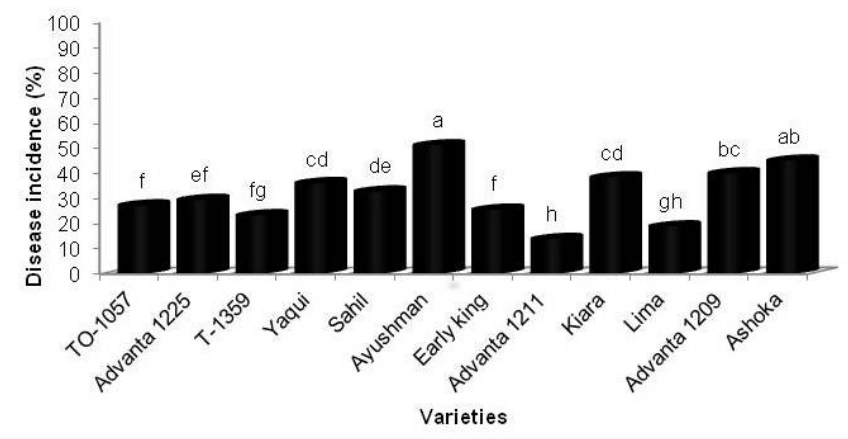

Figure 1: Average incidence of tomato leaf curl disease in different tomato varieties.

The disease appeared at $3^{\text {rd }}$ week after transplantation of seedlings in the variety Advanta-1225, T-1359, Ayushman, Kiara, Advanta-1209 and Ashoka (Table 3 ). The disease was gradually increasing till the time of last assessment. Significantly minimum (38.8\%) incidence of the disease was recorded in variety Advanta-1211 followed by Lima (44.3\%) at $9^{\text {th }}$ and $10^{\text {th }}$ week after transplantation (Table 3). Maximum 89.0 $\%$ disease incidence was recorded in the variety Ayushman and Advanta-1209 followed by Kiara (83.5 $\%)$ at $9^{\text {th }}$ and $10^{\text {th }}$ week after transplantation (Table 3). Whereas variety Yaqui showed 77.8 and $83.3 \%$ incidence of the disease at $9^{\text {th }}$ and $10^{\text {th }}$ week after transplantation, respectively (Table 3 ). 
Table 2: Incidence of tomato leaf curl disease in different tomato varieties during kharif and rabi growing season 2017-2018.

\begin{tabular}{llll} 
Varieties & Season & \multicolumn{2}{l}{ Incidence (\%) } \\
TO-1057 & Kharif & 44.53 & $\mathrm{~b}-\mathrm{e}$ \\
Advanta 1225 & Rabi & 9.90 & $\mathrm{~lm}$ \\
& Kharif & 37.73 & $\mathrm{~d}-\mathrm{h}$ \\
T-1359 & Rabi & 21.10 & $\mathrm{lk}$ \\
& Kharif & 34.50 & $\mathrm{f}-\mathrm{i}$ \\
Yaqui & Rabi & 12.10 & $\mathrm{k}-\mathrm{m}$ \\
& Kharif & 35.57 & $\mathrm{e}-\mathrm{i}$ \\
Sahil & Rabi & 36.67 & $\mathrm{e}-\mathrm{i}$ \\
& Kharif & 47.83 & $\mathrm{bc}$ \\
Ayushman & Rabi & 17.70 & $\mathrm{kl}$ \\
& Kharif & 71.13 & $\mathrm{a}$ \\
Early king & Rabi & 31.13 & $\mathrm{~g}-\mathrm{i}$ \\
& Kharif & 36.73 & $\mathrm{e}-\mathrm{i}$ \\
Advanta 1211 & Rabi & 14.30 & $\mathrm{k}-\mathrm{m}$ \\
& Kharif & 21.10 & $\mathrm{jk}$ \\
Kiara & Rabi & 6.60 & $\mathrm{~m}$ \\
& Kharif & 46.70 & $\mathrm{~b}-\mathrm{d}$ \\
Lima & Rabi & 30.07 & $\mathrm{~h}-\mathrm{j}$ \\
& Kharif & 9.90 & lm \\
Advanta 1209 & Rabi & 27.80 & $\mathrm{ij}$ \\
Ashoka & Kharif & 38.87 & $\mathrm{c}-\mathrm{h}$ \\
\multirow{2}{*}{ LSD (a= 0.05) } & Rabi & 41.17 & $\mathrm{~b}-\mathrm{f}$ \\
& Kharif & 50.13 & $\mathrm{~b}$ \\
& Rabi & 40.00 & $\mathrm{c}-\mathrm{g}$ \\
& $9.0892 \pm 4.6257$ & \\
& & & \\
& & &
\end{tabular}

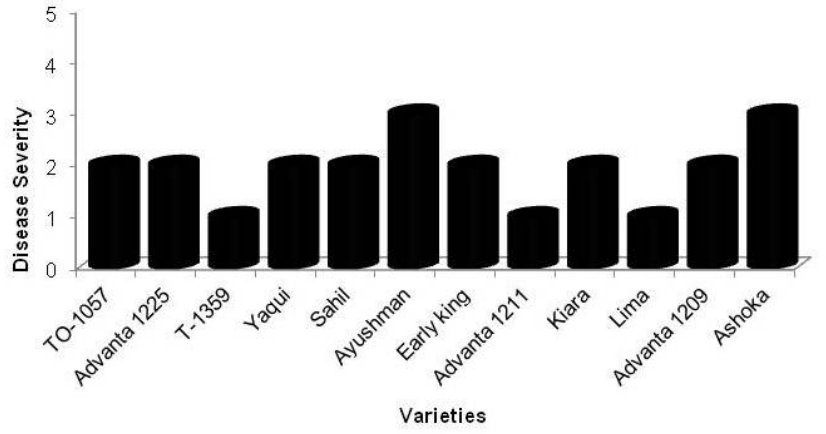

Figure 2: Average severity of tomato leaf curl disease in different tomato varieties.

Severity of the leaf curl disease in different tomato varieties

Severity of the TLCVD was recorded using 0-5 rating scale. Minimum 1-rating score was recorded in T-1359, Advanta-1211 and Lima varieties (Figure 2). Maximum severity score 3 was recorded in Ayushman and Ashoka varieties (Figure 2). All the remaining varieties showed 2-severity score (Figure 2). During rabi growing season severity score- 1 of the disease was recorded in varieties like TO-1057, Advanta-1225, T-1359, Sahil, Early king and Advanta-1211 (Table 4). Yaqui and Advanta-1209 varieties showed severity score- 2 during both growing seasons (Table 4). Ayushman variety showed maximum severity 4-score during kharif growing season followed by TO-1057, Sahil, Kiara and Ashoka variety with 3 -score (Table 4). Severity score-2 was recorded in variety Advanta-1225, T-1359 and Early king during kharif growing season (Table 4). Ayushman, Lima and Ashoka varieties showed 2-score severity during

Table 3: Incidence of tomato leaf curl disease in different tomato varieties over the growth period.

\begin{tabular}{|c|c|c|c|c|c|c|c|c|c|c|}
\hline \multirow[t]{2}{*}{ Varieties } & \multicolumn{10}{|c|}{ Weeks after transplanting } \\
\hline & $1^{\text {st }}$ & $2^{\text {nd }}$ & $3^{\text {rd }}$ & $4^{\text {th }}$ & $5^{\text {th }}$ & $6^{\text {th }}$ & $7^{\text {th }}$ & $8^{\text {th }}$ & $9^{\text {th }}$ & $10^{\text {th }}$ \\
\hline TO-1057 & $0.0 \mathrm{n}$ & $0.0 \mathrm{n}$ & $0.0 \mathrm{n}$ & $16.6 \mathrm{k}-\mathrm{n}$ & $22.1 \mathrm{j}-\mathrm{m}$ & $33.3 \mathrm{~h}-\mathrm{k}$ & $44.5 \mathrm{f}-\mathrm{i}$ & $50.0 \mathrm{e}-\mathrm{h}$ & $50.0 \mathrm{e}-\mathrm{h}$ & $55.5 \mathrm{~d}-\mathrm{g}$ \\
\hline Advanta-1225 & $0.0 \mathrm{n}$ & $0.0 \mathrm{n}$ & $11.01-\mathrm{n}$ & $11.01-n$ & $11.01-n$ & $33.1 \mathrm{~h}-\mathrm{k}$ & $44.5 \mathrm{f}-\mathrm{i}$ & $50.0 \mathrm{e}-\mathrm{h}$ & $61.1 \mathrm{c}-\mathrm{f}$ & $72.3 \mathrm{a}-\mathrm{d}$ \\
\hline $\mathrm{T}-1359$ & $0.0 \mathrm{n}$ & $0.0 \mathrm{n}$ & $5.5 \mathrm{mn}$ & $5.5 \mathrm{mn}$ & $11.11-n$ & $22.1 \mathrm{j}-\mathrm{m}$ & $33.3 \mathrm{~h}-\mathrm{k}$ & $38.8 \mathrm{~g}-\mathrm{j}$ & $55.5 \mathrm{~d}-\mathrm{g}$ & $61.0 c-f$ \\
\hline Yaqui & $0.0 \mathrm{n}$ & $0.0 \mathrm{n}$ & $0.0 \mathrm{n}$ & $11.01-\mathrm{n}$ & $22.1 \mathrm{j}-\mathrm{m}$ & $50.0 \mathrm{e}-\mathrm{h}$ & $50.0 \mathrm{e}-\mathrm{h}$ & $66.8 \mathrm{~b}-\mathrm{e}$ & $77.8 \mathrm{a}-\mathrm{c}$ & $83.3 \mathrm{ab}$ \\
\hline Sahil & $0.0 \mathrm{n}$ & $0.0 \mathrm{n}$ & $0.0 \mathrm{n}$ & $22.1 \mathrm{j}-\mathrm{m}$ & $27.6 \mathrm{i}-1$ & $33.3 \mathrm{~h}-\mathrm{k}$ & $50.0 \mathrm{e}-\mathrm{h}$ & $61.1 \mathrm{c}-\mathrm{f}$ & $66.6 \mathrm{~b}-\mathrm{e}$ & $66.6 \mathrm{~b}-\mathrm{e}$ \\
\hline Ayushman & $0.0 \mathrm{n}$ & $0.0 \mathrm{n}$ & $22.1 \mathrm{j}-\mathrm{m}$ & $39.0 \mathrm{~g}-\mathrm{j}$ & $61.1 \mathrm{c}-\mathrm{f}$ & $66.6 \mathrm{~b}-\mathrm{e}$ & $72.1 \mathrm{a}-\mathrm{d}$ & $72.1 \mathrm{a}-\mathrm{d}$ & $89.0 \mathrm{a}$ & $89.0 \mathrm{a}$ \\
\hline Early king & $0.0 \mathrm{n}$ & $0.0 \mathrm{n}$ & $0.0 \mathrm{n}$ & $0.0 \mathrm{n}$ & $11.01-n$ & $38.8 \mathrm{~g}-\mathrm{j}$ & $38.8 \mathrm{~g}-\mathrm{j}$ & $50.0 \mathrm{e}-\mathrm{h}$ & $55.5 \mathrm{~d}-\mathrm{g}$ & $61.0 \mathrm{c}-\mathrm{f}$ \\
\hline Advanta-1211 & $0.0 \mathrm{n}$ & $0.0 \mathrm{n}$ & $0.0 \mathrm{n}$ & $5.50 \mathrm{mn}$ & $5.5 \mathrm{mn}$ & $5.5 \mathrm{mn}$ & $16.6 \mathrm{k}-\mathrm{n}$ & $27.6 \mathrm{i}-1$ & $38.8 \mathrm{~g}-\mathrm{j}$ & $38.8 \mathrm{~g}-\mathrm{j}$ \\
\hline Kiara & $0.0 \mathrm{n}$ & $0.0 \mathrm{n}$ & $11.11-n$ & $11.11-n$ & $16.6 \mathrm{k}-\mathrm{n}$ & $44.3 \mathrm{f}-\mathrm{i}$ & $61.1 \mathrm{c}-\mathrm{f}$ & $72.3 \mathrm{a}-\mathrm{d}$ & $83.5 \mathrm{ab}$ & $83.5 \mathrm{ab}$ \\
\hline Lima & $0.0 \mathrm{n}$ & $0.0 \mathrm{n}$ & $0.0 \mathrm{n}$ & $0.0 \mathrm{n}$ & $5.5 \mathrm{mn}$ & $22.1 \mathrm{j}-\mathrm{m}$ & $33.3 \mathrm{~h}-\mathrm{k}$ & $38.8 \mathrm{~g}-\mathrm{j}$ & $44.3 \mathrm{f}-\mathrm{i}$ & $44.3 \mathrm{f}-\mathrm{i}$ \\
\hline Advanta-1209 & $0.0 \mathrm{n}$ & $0.0 \mathrm{n}$ & $5.5 \mathrm{mn}$ & $16.5 \mathrm{k}-\mathrm{n}$ & $22.0 \mathrm{j}-\mathrm{m}$ & $44.5 \mathrm{f}-\mathrm{i}$ & $61.3 \mathrm{c}-\mathrm{f}$ & $72.3 \mathrm{a}-\mathrm{d}$ & $89.0 \mathrm{a}$ & $89.0 \mathrm{a}$ \\
\hline Ashoka & $0.0 \mathrm{n}$ & $0.0 \mathrm{n}$ & $11.11-n$ & $27.8 \mathrm{i}-1$ & $44.3 \mathrm{f}-\mathrm{i}$ & $55.6 \mathrm{~d}-\mathrm{g}$ & $72.3 \mathrm{a}-\mathrm{d}$ & $72.3 \mathrm{a}-\mathrm{d}$ & $83.5 \mathrm{ab}$ & $83.5 \mathrm{ab}$ \\
\hline $\operatorname{LSD}(a=0.05)$ & \multicolumn{10}{|c|}{$20.324 \pm 10.343$} \\
\hline
\end{tabular}


Table 4: Severity of tomato leaf curl disease in different tomato varieties during kharif and rabi growing season 2017-2018.

\begin{tabular}{lll} 
Varieties & Season & Severity \\
TO-1057 & Kharif & 3 \\
Advanta 1225 & Rabi & 1 \\
\multirow{2}{*}{ T-1359 } & Kharif & 2 \\
& Rabi & 1 \\
Yaqui & Kharif & 2 \\
Sahil & Rabi & 1 \\
& Kharif & 2 \\
Ayushman & Rabi & 2 \\
& Kharif & 3 \\
Early king & Rabi & 1 \\
& Kharif & 4 \\
Advanta 1211 & Rabi & 2 \\
& Kharif & 2 \\
Kiara & Rabi & 1 \\
& Kharif & 1 \\
Lima & Rabi & 1 \\
& Kharif & 3 \\
Advanta 1209 & Rabi & 2 \\
Ashoka & Kharif & 1 \\
& Rabi & 2 \\
& Kharif & 2 \\
& Rabi & 2 \\
& Kharif & 3 \\
& Rabi & 2 \\
& &
\end{tabular}

Table 5: Severity of tomato leaf curl disease in different tomato varieties over the growth period.

Varieties Weeks after transplanting $1^{\text {st }} \quad 2^{\text {nd }} \quad 3^{\text {th }} \quad 4^{\text {th }} \quad 5^{\text {th }} 6^{\text {th }} 7^{\text {th }} 8^{\text {th }} 9^{\text {th }} \quad 10^{\text {th }}$

TO-1057

$\begin{array}{llllllllll}0.0 & 0.0 & 0.0 & 1 & 1 & 2 & 3 & 3 & 3 & 3\end{array}$

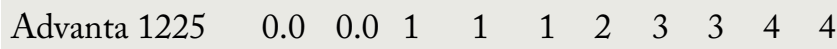

$\begin{array}{lllllllllll}\mathrm{T}-1359 & 0.0 & 0.0 & 1 & 1 & 1 & 1 & 2 & 2 & 3 & 3\end{array}$

Yaqui $\quad \begin{array}{lllllllllll}0.0 & 0.0 & 0.0 & 1 & 1 & 3 & 3 & 4 & 4 & 4\end{array}$

Sahil $\quad \begin{array}{lllllllllll}0.0 & 0.0 & 0.0 & 1 & 2 & 2 & 3 & 3 & 4 & 4\end{array}$

Ayushman $\quad \begin{array}{lllllllllll}0.0 & 0.0 & 1 & 2 & 3 & 4 & 4 & 4 & 5 & 5\end{array}$

Early king $\quad \begin{array}{lllllllllll}0.0 & 0.0 & 0.0 & 0.0 & 1 & 2 & 2 & 3 & 3 & 3\end{array}$

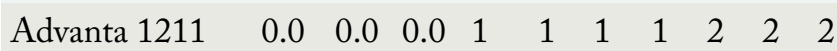

Kiara $\quad \begin{array}{lllllllllll}0.0 & 0.0 & 1 & 1 & 1 & 3 & 4 & 4 & 5 & 5\end{array}$

Lima $\quad \begin{array}{lllllllllll}0.0 & 0.0 & 0.0 & 0.0 & 1 & 2 & 2 & 2 & 3 & 3\end{array}$

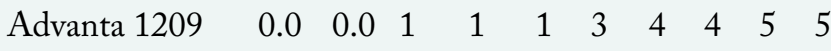

$\begin{array}{lllllllllll}\text { Ashoka } & 0.0 & 0.0 & 1 & 2 & 3 & 3 & 4 & 4 & 4 & 4\end{array}$ rabi season (Table 4). During kharif growing season minimum 1-score of the severity was recorded in variety Advanta-1211 and Lima (Table 4). Kiara variety during rabi season showed 2-score of the severity ( $\mathrm{Ta}-$ ble 4). Similar as the disease incidence, severity of the disease was also recorded in $3^{\text {rd }}$ week after transplantation in few varieties such as Advanta-1225, T-1359, Ayushman, Kiara which were showing 1sverity score (Table 5). Severity of the disease showed increase till $9^{\text {th }}$ and $10^{\text {th }}$ week of observation, however, at last assessment of the disease variety Advanta-1211 showed minimum 2-score whereas maximum 5-score was recorded in Ayushman, Kiara and Advanta-1209 varieties (Table 5). Varieties such as TO-1057, T-1359, Early king and Lima showed 3-severity score at $10^{\text {th }}$ week after transplantation and remaining Advanta-1225, Yaqui, Sahil and Ashoka showed 4 severity score (Table 5).

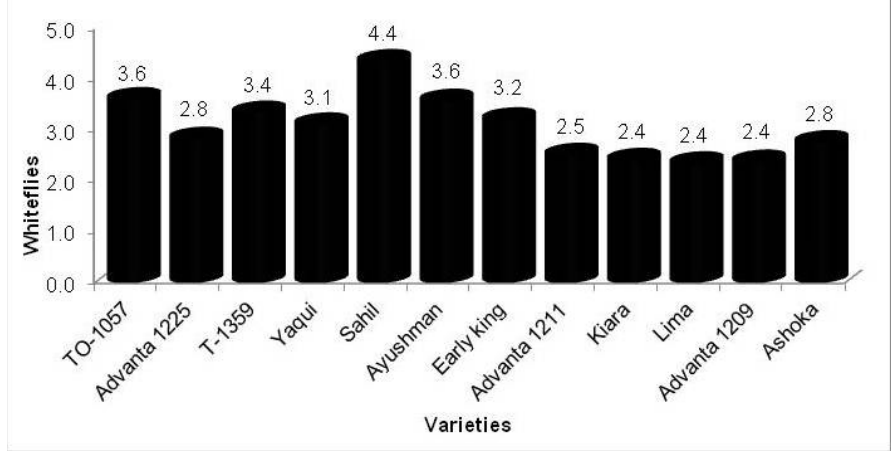

Figure 3: Average whiteflies population in different tomato varieties.

The vector population in different tomato varieties and its correlation with tomato leaf curl viral disease incidence and severity

Maximum vector population was recorded in variety Sahil (4.3 whiteflies) followed by TO-1057 (3.6 whiteflies), Ayushman (3.6 whiteflies), T-1359 (3.3 whiteflies), Early king (3.2 whiteflies), Yaqui (3.1 whiteflies), Advanta-1225 (2.8 whiteflies) and Ashoka (2.8 whiteflies) (Figure 3). Significantly minimum number of whiteflies was recorded in variety Lima (2.3 whiteflies), Advanta-1209 (2.3 whiteflies), Kiara (2.4 whiteflies) followed by Advanta-1211 (2.5 whiteflies) (Figure 3). Maximum vector population (5.9 whiteflies) was recorded in Sahil variety followed by TO-1057 (5.0 whiteflies), Ayushman (4.2 whiteflies) during kharif growing season (Table 6). Whereas minimum vector population was recorded in variety Lima (1.8 whiteflies) during rabi season (Table 6). Maximum 5.7 whiteflies were recorded at $10^{\text {th }}$ week after transplantation in variety Ayushman followed by 
Table 6: Whiteflies population in different tomato varieties during kharif and rabi growing season 2017-2018.

\begin{tabular}{|c|c|c|c|}
\hline \multirow{2}{*}{$\begin{array}{l}\text { Varieties } \\
\text { TO-1057 }\end{array}$} & \multirow{2}{*}{$\begin{array}{l}\text { Season } \\
\text { Kharif }\end{array}$} & \multicolumn{2}{|c|}{ Whiteflies } \\
\hline & & 5.0 & $\mathrm{ab}$ \\
\hline \multirow{3}{*}{ Advanta 1225} & Rabi & 2.2 & $\mathrm{f}$ \\
\hline & Kharif & 3.3 & $c-f$ \\
\hline & Rabi & 2.3 & $d-f$ \\
\hline \multirow[t]{2}{*}{ T-1359 } & Kharif & 3.8 & $b-d$ \\
\hline & Rabi & 2.9 & $c-f$ \\
\hline \multirow[t]{2}{*}{ Yaqui } & Kharif & 3.7 & b-e \\
\hline & Rabi & 2.4 & $d-f$ \\
\hline \multirow[t]{2}{*}{ Sahil } & Kharif & 5.8 & a \\
\hline & Rabi & 2.8 & $c-f$ \\
\hline \multirow[t]{2}{*}{ Ayushman } & Kharif & 4.2 & $\mathrm{bc}$ \\
\hline & Rabi & 2.9 & $c-f$ \\
\hline \multirow[t]{2}{*}{ Early king } & Kharif & 2.1 & $\mathrm{f}$ \\
\hline & Rabi & 4.2 & $\mathrm{bc}$ \\
\hline \multirow[t]{2}{*}{ Advanta 1211} & Kharif & 2.2 & ef \\
\hline & Rabi & 2.7 & $c-f$ \\
\hline \multirow[t]{2}{*}{ Kiara } & Kharif & 2.3 & $d-f$ \\
\hline & Rabi & 2.5 & $d-f$ \\
\hline \multirow[t]{2}{*}{ Lima } & Kharif & 1.8 & $\mathrm{f}$ \\
\hline & Rabi & 2.9 & $c-f$ \\
\hline \multirow[t]{2}{*}{ Advanta 1209} & Kharif & 2.5 & $d-f$ \\
\hline & Rabi & 2.2 & $\mathrm{f}$ \\
\hline \multirow[t]{2}{*}{ Ashoka } & Kharif & 2.6 & $d-f$ \\
\hline & Rabi & 2.9 & $c-f$ \\
\hline $\operatorname{LSD}(a=0.05)$ & \multicolumn{3}{|c|}{$1.5190 \pm 0.7731$} \\
\hline
\end{tabular}

$8^{\text {th }}$ week in variety Early King (5.1) and $3^{\text {rd }}$ week after transplantation in variety T-1359 (Table 7). Whereas minimum (0.5) vector population was recorded at $2^{\text {nd }}$ week after transplantation in Kiara variety (Table 7 ).

Relationships of vector population with incidence and severity of TLCVD was determined using PRISM v. 5.01 (Graph Pad Software). There was non-significant relationship of vector insect with both incidences as well severity of tomato leaf curl disease, $r^{2}=0.07068, P=0.8272$ and $r^{2}=0.2869, P=0.3660$, respectively (Table 8).

The disease appeared at $3^{\text {rd }}$ week after transplantation of seedlings in the variety Advanta-1225, T-1359, Ayushman, Kiara, Advanta-1209 and Ashoka. The disease was gradually increasing till the last assessment. Such trend has been observed in other vector transmitted plant viral diseases (Khoso et al., 2021). These results are accordance with Zhang et al. (2008) who observed that first symptom of the disease can be appear 15 days after transplantation, which are related with longer period till the end of crop. The minimum incidence of the disease was recorded in variety Advanta-1211 (13.85\%) and Lima (18.85\%) followed by $\mathrm{T}-1359$ (23.30\%), Early king (25.51\%), TO-1057 (27.21\%), Advanta-1225 (29.41\%). Whereas minimum 1-rating score was recorded in T-1359, Advanta-1211 and Lima varieties. Generally, the disease incidence was higher in kharif as compare to rabi season but in variety Yaqui, Lima and Advanta-1209 the situation was vice versa. This season variation in the

Table 7: Whitefies population in different tomato varieties over the growth period.

Varieties Weeks after transplanting

$\begin{array}{lllllllllll} & 1^{\text {st }} & 2^{\text {nd }} & 3^{\text {rd }} & 4^{\text {th }} & 5^{\text {th }} & 6^{\text {th }} & 7^{\text {th }} & 8^{\text {th }} & 9^{\text {th }} & 1^{\text {th }} \\ \text { TO-1057 } & 2.0 \mathrm{c}-\mathrm{k} & 4.6 \mathrm{~b}-\mathrm{f} & 4.0 \mathrm{~b}-\mathrm{i} & 3.8 \mathrm{~b}-\mathrm{j} & 4.5 \mathrm{~b}-\mathrm{f} & 2.5 \mathrm{~b}-\mathrm{k} & 3.8 \mathrm{~b}-\mathrm{j} & 5.0 \mathrm{~b}-\mathrm{d} & 3.0 \mathrm{~b}-\mathrm{k} & 2.8 \mathrm{~b}-\mathrm{k} \\ \text { Advanta } 1225 & 1.6 \mathrm{~d}-\mathrm{k} & 2.0 \mathrm{c}-\mathrm{k} & 2.6 \mathrm{~b}-\mathrm{k} & 2.5 \mathrm{~b}-\mathrm{k} & 3.6 \mathrm{~b}-\mathrm{j} & 3.0 \mathrm{~b}-\mathrm{k} & 2.8 \mathrm{~b}-\mathrm{k} & 3.6 \mathrm{~b}-\mathrm{j} & 2.3 \mathrm{~b}-\mathrm{k} & 4.0 \mathrm{~b}-\mathrm{i} \\ \text { T-1359 } & 2.1 \mathrm{c}-\mathrm{k} & 2.5 \mathrm{~b}-\mathrm{k} & 5.1 \mathrm{bc} & 3.6 \mathrm{~b}-\mathrm{j} & 4.6 \mathrm{~b}-\mathrm{f} & 4.1 \mathrm{~b}-\mathrm{h} & 3.8 \mathrm{~b}-\mathrm{j} & 2.8 \mathrm{~b}-\mathrm{k} & 2.1 \mathrm{c}-\mathrm{k} & 2.3 \mathrm{~b}-\mathrm{k} \\ \text { Yaqui } & 2.0 \mathrm{c}-\mathrm{k} & 2.5 \mathrm{~b}-\mathrm{k} & 3.8 \mathrm{~b}-\mathrm{j} & 3.3 \mathrm{~b}-\mathrm{k} & 3.6 \mathrm{~b}-\mathrm{j} & 3.1 \mathrm{~b}-\mathrm{k} & 3.8 \mathrm{~b}-\mathrm{j} & 3.8 \mathrm{~b}-\mathrm{j} & 2.3 \mathrm{~b}-\mathrm{k} & 2.6 \mathrm{~b}-\mathrm{k} \\ \text { Sahil } & 11.6 \mathrm{a} & 2.5 \mathrm{~b}-\mathrm{k} & 2.8 \mathrm{~b}-\mathrm{k} & 4.8 \mathrm{~b}-\mathrm{e} & 3.1 \mathrm{~b}-\mathrm{k} & 3.8 \mathrm{~b}-\mathrm{j} & 3.3 \mathrm{~b}-\mathrm{k} & 2.8 \mathrm{~b}-\mathrm{k} & 3.8 \mathrm{~b}-\mathrm{j} & 4.8 \mathrm{~b}-\mathrm{e} \\ \text { Ayushman } & 0.8 \mathrm{~h}-\mathrm{k} & 1.8 \mathrm{c}-\mathrm{k} & 4.3 \mathrm{~b}-\mathrm{g} & 3.1 \mathrm{~b}-\mathrm{k} & 4.6 \mathrm{~b}-\mathrm{f} & 3.3 \mathrm{~b}-\mathrm{k} & 3.8 \mathrm{~b}-\mathrm{j} & 4.1 \mathrm{~b}-\mathrm{h} & 4.0 \mathrm{~b}-\mathrm{i} & 5.6 \mathrm{~b} \\ \text { Early king } & 0.8 \mathrm{~h}-\mathrm{k} & 1.3 \mathrm{f}-\mathrm{k} & 3.0 \mathrm{~b}-\mathrm{k} & 2.8 \mathrm{~b}-\mathrm{k} & 3.3 \mathrm{~b}-\mathrm{k} & 4.1 \mathrm{~b}-\mathrm{h} & 3.1 \mathrm{~b}-\mathrm{k} & 5.1 \mathrm{~b}-\mathrm{c} & 4.3 \mathrm{~b}-\mathrm{g} & 4.0 \mathrm{~b}-\mathrm{i} \\ \text { Advanta 1211 } & 0.6 \mathrm{i}-\mathrm{k} & 1.3 \mathrm{f}-\mathrm{k} & 3.5 \mathrm{~b}-\mathrm{j} & 2.3 \mathrm{~b}-\mathrm{k} & 3.0 \mathrm{~b}-\mathrm{k} & 3.3 \mathrm{~b}-\mathrm{k} & 2.6 \mathrm{~b}-\mathrm{k} & 2.8 \mathrm{~b}-\mathrm{k} & 2.3 \mathrm{~b}-\mathrm{k} & 3.1 \mathrm{~b}-\mathrm{k} \\ \text { Kiara } & 1.0 \mathrm{~g}-\mathrm{k} & 0.5 \mathrm{j}-\mathrm{k} & 2.0 \mathrm{c}-\mathrm{k} & 2.5 \mathrm{~b}-\mathrm{k} & 3.0 \mathrm{~b}-\mathrm{k} & 2.7 \mathrm{~b}-\mathrm{k} & 3.8 \mathrm{~b}-\mathrm{j} & 3.3 \mathrm{~b}-\mathrm{k} & 2.8 \mathrm{~b}-\mathrm{k} & 2.5 \mathrm{~b}-\mathrm{k} \\ \text { Lima } & 0.0 \mathrm{k} & 0.7 \mathrm{i}-\mathrm{k} & 1.7 \mathrm{~d}-\mathrm{k} & 2.3 \mathrm{~b}-\mathrm{k} & 2.8 \mathrm{~b}-\mathrm{k} & 3.7 \mathrm{~b}-\mathrm{j} & 4.1 \mathrm{~b}-\mathrm{h} & 3.3 \mathrm{~b}-\mathrm{k} & 1.8 \mathrm{c}-\mathrm{k} & 3.0 \mathrm{~b}-\mathrm{k} \\ \text { Advanta 1209 } & 0.8 \mathrm{~h}-\mathrm{k} & 2.1 \mathrm{c}-\mathrm{k} & 2.7 \mathrm{~b}-\mathrm{k} & 2.8 \mathrm{~b}-\mathrm{k} & 2.1 \mathrm{c}-\mathrm{k} & 2.7 \mathrm{~b}-\mathrm{k} & 3.0 \mathrm{~b}-\mathrm{k} & 3.0 \mathrm{~b}-\mathrm{k} & 3.0 \mathrm{~b}-\mathrm{k} & 1.5 \mathrm{e}-\mathrm{k} \\ \text { Ashoka } & 1.3 \mathrm{f}-\mathrm{k} & 2.7 \mathrm{~b}-\mathrm{k} & 3.0 \mathrm{~b}-\mathrm{k} & 3.1 \mathrm{~b}-\mathrm{k} & 3.7 \mathrm{~b}-\mathrm{j} & 4.0 \mathrm{~b}-\mathrm{i} & 2.3 \mathrm{~b}-\mathrm{k} & 2.0 \mathrm{c}-\mathrm{k} & 2.3 \mathrm{~b}-\mathrm{k} & 3.1 \mathrm{~b}-\mathrm{k} \\ \text { LSD (a=0.05) } & 3.3966 \pm 1.7286 & & & & & & & & \end{array}$


intensity of the TLCVD is attributed with higher temperature range (Iftkhar et al., 2021). In the geographic area of current study temperature remains higher in the kharif growing season.

Table 8: Relationships of vector population with intensity of tomato leaf curl disease.

$\begin{array}{lll}\text { Particular of analysis } & \begin{array}{l}\text { Disease inci- } \\ \text { dence (\%) }\end{array} & \text { Disease severity } \\ \text { Spearman r } & 0.07068 & 0.2869 \\ \text { 95\% confidence interval } & -0.5385 \text { to } 0.6313 & -0.3607 \text { to } 0.7478 \\ \text { P } & 0.8272 & 0.3660 \\ \text { a } & 0.05 & \end{array}$

These results are in accordance with Channarayappa and Shivashank (1992) screened 1200 cultivars, breeding lines and wild species of tomato for resistance to tomato leaf curl disease, which all were less or more susceptible to the disease, one three lines of $L$. hirsutum and one of L. peruvianum accessions showed apparent resistance to the disease. Similarly, Hameed and Khalid (1996) collected tomato cultivars from AVRDC for screening resistance to TLCV, only Tyking (a Hybrid cultivar), L. hirsutum (LA-1777) and L. chilenese (LA-1969) are resistant to the virus. Singh (2014) screened 32 tomato genotypes against TLCVD under greenhouse out of which three genotypes showed resistant reaction and eight genotypes found moderately resistant. Zeshan et al.(2016) in their study concluded that number of highly susceptible, susceptible, moderately susceptible, moderately resistant and resistant to TLCVD were three, six, four, six and eight, respectively. Highly resistant and immune varieties/ lines against TLCVD were not found in the study.

Maximum vector population was recorded in variety Sahil followed by TO-1057, Ayushman, T-1359, Early king, Yaqui, Advanta-1225 and Ashoka Significantly minimum number of whiteflies was recorded in variety Lima, Advanta-1209, Kiara followed by Advanta-1211. There was non-significant relationship of vector insect with both incidences as well severity of tomato leaf curl disease, $r^{2}=0.07068, P=0.8272$ and $r^{2}=0.2869, P=0.3660$, respectively. Such findings are previously reported by Ssekyewa et al. (2007) who assessed a negative relationship between number of plants infected with tomato yellow leaf curl virus and percentage marketable tomato yield. Tomato maturity was inversely proportional to whitefly infestation. Later on, Ssekyewa et al. (2010) reported that inci- dence of tomato yellow leaf curl disease was lower, whereas the vector population was higher at seedling stage. It is well documented that a single individual of insect vector can transmit the virus to a single or dozen of susceptible plants. Under circumstance single individual of insect Therefore under this circumstance non-significant variation might be associated with such characteristic of whitefly that single individual of whitefly may sufficiently transmit the TLCV to susceptible tomato plant. However, further studies are needed to explore this phenomenon.

\section{Conclusions and Recommendations}

Based on the obtained results it is concluded that the minimum incidence and severity of tomato leaf curl disease was recorded in variety Advanta-1211, Lima and $\mathrm{T}-1359$. In rabi season the disease intensity was lower as compare to kharif season. Maximum vector population was recorded in variety Sahil followed by TO-1057, Ayushman, T-1359, Early king, Yaqui, Advanta-1225 and Ashoka. The disease intensity was not dependent to the vector population. Therefore, these varieties should incorporate in the disease management plan. Same set of varieties should also be tested under challenged inoculation and different isolates may test for more authenticate the results. Impact of environmental conditions may be studies for furthermore in detail to understate the epidemic parameters of the disease.

\section{Acknowledgments}

This work is part of master's thesis submitted to department of Plant Pathology, Faculty of Crop Protection, Sindh Agriculture University, Tandojam 70060, Pakistan.

\section{Author's Contribution}

Sajad Ali Solangi: Performed Experiments and collected data.

Jamal-U-Ddin Hajano, Rehana Naz Syed, Sohail Ahmed Otho, Fahad Nazir Khoso, Agha Mushtaque Ahmed and Khadim Hussain Wagan: Performed Analysis.

\section{Sajad Ali Solangi, Suman Tarique Qazi and Aftab}

Raza Jarwar: Wrote first draft of the manuscript.

All authors contributed to the study conception and design and commented on final versions of the man- 
uscript. All authors read and approved the final manuscript.

\section{Conflict of interest}

The authors have declared no conflict of interest.

\section{References}

Akram, M.F., Hafeez, M. Farooq, M. Arshad, M. Hussain, S. Ahmed, K. Zia and A. A.K. Hafiz. 2013. A case to study population dynamics of Bemisia tabaci and Thrips tabaci on $B t$ and non- $B t$ cotton genotypes. Pak. J. Agri. Sci., 50: 617-623.

Brunt, A.A., K. Crabtree, M.J. Dallwitz, A.J. Gibbs, L. Watson and E.J. Zurcher. (eds.) 1996. Plant Viruses Online: Descriptions and Lists from the VIDE Database. Version: $20^{\text {th }}$ August 1996.' URL http://biology.anu.edu.au/Groups/ MES/vide/

Channarayappa, G. and Shivashank. 1992. Resistance of Lycopersicon species to Bemisia tabaci, a tomato leaf curl virus vector. Can.J. Bot., 70(11): 2184-2192. https://doi.org/10.1139/ b92-270

Dallwitz, M.J. 1980. A general system for coding taxonomic descriptions. Taxon, 29:41-46. https://doi.org/10.2307/1219595

GoP. 2018. Pakistan Statistical Yearbook. Government of Pakistan, Ministry of Statistics, Statistics Division.

Hameed, S. and S. Khalid. 1996. Screening of tomato lines/varieties against tomato leaf curl virus. Crop Prot. Conf., Apr. 20-22, 1996, NWFP Agri. Univ. Peshawar. pp. 39.

Hassan, S. and P.E. Thomas. 1985. Attenuation and symptoms amelioration of tomato yellow top virus isolates. Sarhad J. Agric., 1(2):243-249.

Hassan, S.M.A. and T. Defoer. 1993. Epidemiological studies of tomato viruses in Malakand Agency of North West Frontier Province, Pakistan. Sarhad J. Agric., 9(1):37-44.

Iftikhar, Y., M. Mubeen, A. Sajid, M.A. Zeshan, Q. Shakeel, A. Abbas, S. Bashir, M. Kamran and H. Anwaar. 2021. Effects of tomato leaf curl virus on growth and yield parameters of tomato crop. Arab. J. Plant Prot., 39(1): 79-83.

Khoso, S., J. Hajano, A.A. Lakho, M.A. Abro, K.H. Dhiloo, K.H. Wagan, F.N. Khoso, A.R. Jarwar,
S.T. Qazi and S. Rizwan. 2021. Screening of okra varieties for resistance to okra yellow vein mosaic disease under field conditions. Int. J. phytopathol., 10(1): 37-48. https://doi. org/10.33687/phytopath.010.01.3516

Nono-womdim, R. 2001. An overview of major virus diseases of vegetable crops in Africa and some aspects of their control. P1. Virol. Sub Sah. Afri., 213-230.

Perveen, R., M.A. Khan, N.U. Islam, S. Chohan, S. Haider and I.A. Nasir. 2010. Whitefly population on different cotton varieties in Punjab. Sarhad J. Agric., 26(4):583-589.

Sadaf, J.Q. M. Khan, U.R. Habib and B. Imam. 2012. Response of tomato yield and postharvest life to potash levels. Sarhad J. Agric., 28(2):227-235.

Shelat, M., S. Murari, M.C. Sharma, R.B. Subramanian, J. Jummanah and B. Jarullah. 2014. Prevalence and distribution of tomato leaf curl virus in major agro climatic zones of Gujarat. Adv. Biol. Sci. Biol. Technol., 5:1-3. https://doi.org/10.4236/abb.2014.51001

Singh, K. 2014. Evaluation of tomato genotypes and its reaction against ToLCV causing leaf curl disease in tomato (Solanum lycopersicon L.) J. Exp. Biol. Agric. Sci., 2(1S):120-125.

Ssekyewa, C., P.L. Van Damme and M. Hofte. 2007. Relationship between tomato yellow leaf curl viruses and the whitefly vector. Common. Agric. Appl. Biol. Sci., 72(4):1029-1048.

Ssekyewa, C., P.V. Damme, F. Nagawa, S. Kyamanywa and K.A. Steele.2010. Relationship between tomato yellow leaf curl viruses and the whitefly vector. J. Sci. Sustain. Dev., 3(1): 7-8. https://doi.org/10.4314/jssd.v3i1.67750

Walkey, D. 1985. Applied Plant Virology, Heinemann; London. pp.6-92.

Zeshan, M.A., M.A. Khan, S. Ali and M. Arshad. 2016. Phenotypic evaluation of tomato germplasm for the source of resistance against tomato leaf curl virus disease. J. Anim. Plant Sci., 26(1):194-200.

Zhang, Y.P., W.M. Zhu, H.M. Cui, Y. Qiu, K. Sha, Y.H. Wan, L.Y. Zhu, L. Yu and Z. Hui. 2008. Molecular identification and the complete nucleotide sequence of TLCV isolate from Shanghai of China. Virol. Gen., 36:547-551. https://doi.org/10.1007/s11262-008-0226-0 